\title{
Impact of genetically nonlinear application of external loads on the stress-strain state and on the principal vibration modes of reinforced concrete frame buildings on elastic subsoil
}

\author{
Oleg Pakhmurin ${ }^{1}$, Victor Mikhaylov², and Matvey Khamgushkeev ${ }^{3, *}$ \\ ${ }^{1}$ Prof. TSUAB, PhD in Engineering Science, 634003 Tomsk, Russia \\ ${ }^{2}$ Head of SCAD SOFT Scientific and Technical Support Centre, MSc, 630132 Novosibirsk, Russia \\ ${ }^{3}$ Engineer, MSc, 634003 Tomsk, Russia
}

\begin{abstract}
In this paper authors discuss the impact of genetically nonlinear application of external loads on the stress-stain state and on principal vibration modes of reinforced concrete frame structures on elastic foundation. The reader will find a methodology for integrated numerical and instrumental analysis. Six examples of linear and nonlinear FEAmodels calculated in SCAD Office are given in comparison.
\end{abstract}

One of the elements that ensure the spatial rigidity of the building frame is the stiffening diaphragm. Currently, the shear wall design is not regulated sufficiently by design codes, for instance, in determining the location of diaphragms on the building plans [1].

Assessment of the static stiffness of a building or a structure can be performed as well on the basis of dynamic characteristics [3]. Analysis of the eigenvalues in the frequency domain or of the principal periods extends extensively the information about the dynamic properties of the structure with the use of FEA-software as SCAD Office [2]. Such analysis would make it possible to make the constructive solutions more reasonable and would reduce the number of iterations needed for the optimal placement of stiffness diaphragms.

When designing multi-storey reinforced concrete frame buildings, two complex tasks arise. The first task is related to the need to ensure sufficient rigidity of the building for its comfortable and safe operation. This problem is solved by the correct location of shear walls in the floor plan and its geometry optimization. The quality of such optimization could be checked by performing a modal analysis of the design model. At the same time, a second problem that arises, is to take into account the genetic nonlinearity of statically indeterminable systems, such as multi-storey reinforced concrete frames. The civil engineers in their traditional practice of computational analysis quite often do not take into account the conditions of the building multistage existence, when its parameters can significantly change in time. For example, such parameters as external loads, constraints or the subsoil modulus of elasticity, etc. The constructed facilities, despite the high level of responsibility and long lifecycle duration, never reside in frozen conditions. Over the entire

\footnotetext{
*Corresponding author: hppynewfear@mail.ru
} 
period of operation, they are subject to many fundamental changes. The construction of a multi-storey structure is obviously a multi-stage process. During this process the erection of a structure is carried out by building up the structure from flour to flour, gradually forming a complete system of supporting constraints, loads and rigidities. Modern FEA-software can easily perform an adequate forecast of the stress-strain state accounting erection stages. The important point here, will be the fact that each subsequent stage of the structure inherits the internal efforts and deformations that were implemented in the previous stages. Consideration of the loading history is significant in buildings with a significant share of self-weight in the total combination of the design loads [4]. The traditional FEA-calculation of the structure with one-stage simultaneous assignment of all external loads can give the calculation results not only quantitatively, but also qualitatively significantly different from the real stress-strain state.

As an example of the incorrect design of the rigidity of a high-rise building frame, the authors cite the consequences in an emergency state seventeen-story residential building, based on to the series of standard designs "KUB 2.5" with extensive torsional displacements and non-bearing wall cracks (Fig. 1). On this example the reader will see how the genetically non-linear loads assignment affects the principal modes and periods, and the stress-strain state of structures on the rigid and elastic subsoils. Such accurate multistorey building modelling is essential for correct shear wall placement.
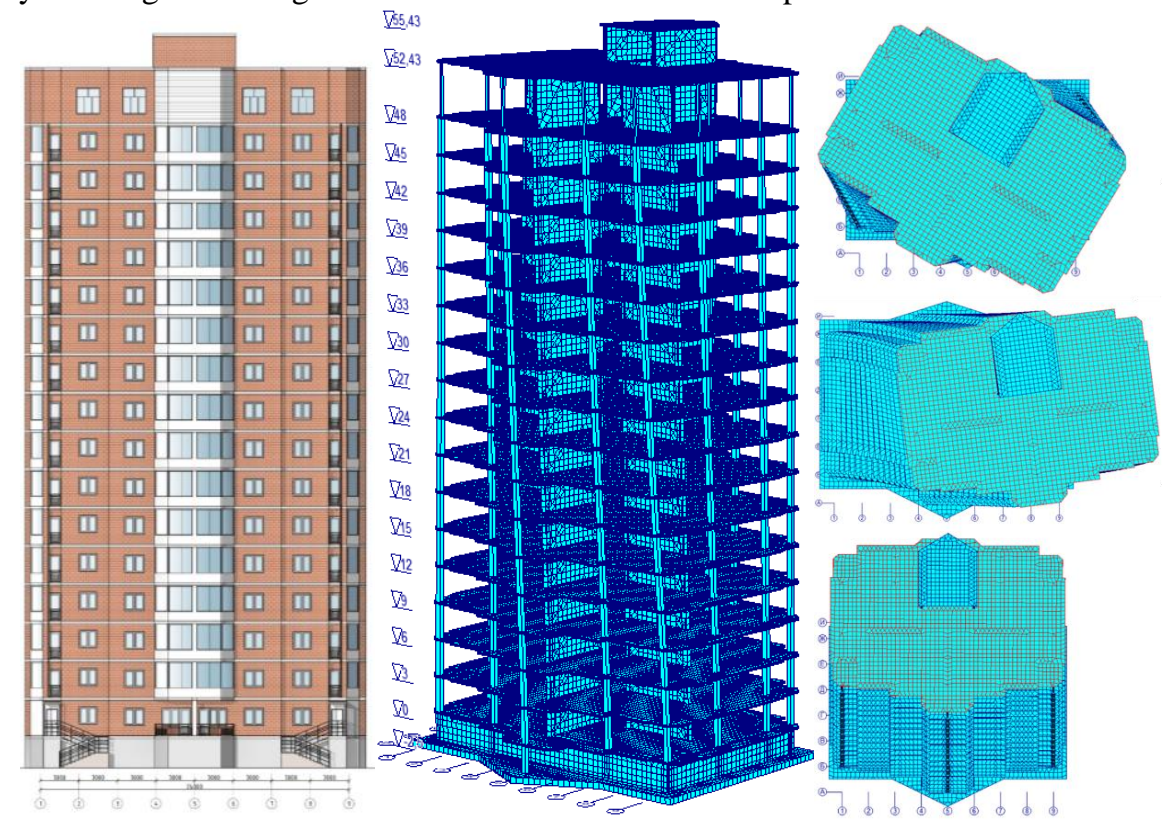

Fig. 1. Analysed multi-storey residential building on the basis of standard design series "KUB 2.5" and its three principal modes of natural oscillations (top down).

Quite often, design engineers relying on expert judgment make mistakes of placement of stiffening diaphragms in the building plan. At the same time, the stiffness diaphragms analysis on the basis of static ultimate strains is fairly accurate and simple. The number and dimensions of the diaphragms cross sections are optimized accounting rigidity and ultimate horizontal deflections for multi-storey buildings. It is recommended that a geometrically irregular structural system should be designed so that the center of rigidity and the center of mass are as close as possible to the location of the resulting vertical load.

The quality of the preliminary static shear wall can be improved by additional analysis of the dynamic characteristics of a multi-storey reinforced concrete frame as for the 
cantilever dynamic model. To ensure the spatial rigidity of a building, the initial task is to determine the ultimate strains of the preliminary calculated diaphragms in the following sequence: a) calculating the total bending moment from the wind at the bottom of the foundation slab, since this an effort that determines the diaphragms rigidity; b) determining the number of diaphragms, and the dimensions of the diaphragm cross-sections by flexural rigidity and ultimate horizontal strains as for high-rise buildings. Since the values of normative wind loads are obtained on different floors, and the main vibration tone depends on the mass distribution and on the rigidity of the structure, the solution should be converted into the simplest console with a flat cross-section of complex shape, and find the total bending moment at the bottom of the foundation slab. Then bending stiffness of one diaphragm is determined. It is defined as well as for the concrete columns. After that, one should calculate the ultimate bending moment for one diaphragm according to the condition of ultimate horizontal strains of the top of the wall from the console flexure formula, as well as the maximum displacements of the top of the diaphragm. Despite the convenience of an analytical solution only for simple single-mass systems, this approach allows us to compare the period of natural oscillations when taking into account flexural or torsional stiffness to ensure that the first two basic forms of natural vibrations of a system has to be flexural and the third form has to be torsional. This requirement, according to the design code SP 14.13330.2018 "Construction in seismic regions", allows one to analyze the seismic effects only in two directions for building models with the simple regular geometry.

After completing the preliminary calculation of the diaphragms rigidity, taking into account the ultimate strains, and ensuring the first two bending modes of natural vibrations, as well as dynamically comfortable accelerations, the authors recommend to perform a modal analysis of the detailed spatial dynamic model. Such FEA-model of the building has a higher degree of reliability, in contrast to the analytical calculations of the building as for a single-mass console dynamic model. In addition, the authors recommend to compare the principal periods of natural oscillations of the design model, calculated in modal analysis, with the allowable of the principal periods given in the Appendix G of GOST 34081-2017 "Buildings and Structures. Determining the parameters of the principal modes of natural oscillations". One should take into account the fact that in order to increase the reliability of the principal period FEA calculation, it is necessary to take into account the elasticity of the subsoil.

The second part of the research is based on a comparative analysis of the stress-strain state of a multi-storey reinforced concrete frame building for the results of three linear and three genetically non-linear calculations. In each formulation, the design FEA-model is considered on an absolutely rigid basis; with a design model on a Winkler elastic subsoil of constant rigidity. Additionally, the last comparison describes the subsoil dynamic modulus of elasticity assignment at the last stage of erection and nonlinear loads assignment. The design models are compared by the longitudinal internal forces in the most stressed column of the first floor and by the accelerations on the level of the sixteenth floor. This column could be found by analyzing the energy pre-processor mode in the SCAD FEA-software, which determines the most loaded finite elements. In finite bar element of the column No. 7 the results were obtained from design and long-term effects, and in column No. 20 from seismic impacts.

On the basis of the conducted analytical studies to ensure the required spatial rigidity of the building, the values of the natural oscillation periods have been adopted to the required values in the Appendix G of GOST 34081-2017 by modification of the shear wall geometry. As a result, the maximum displacements of the building frame from wind impacts were also reduced below the ultimate values.

The internal efforts considered in the column from the normative long-term loads under the usual operation conditions on an absolutely rigid subsoil (design model No.1.1 in Fig.2) are less by $20 \%$ than the longitudinal force that could be calculated on an elastic subsoil 
based on the total strain modulus (model No.1.2 in Fig.2). When substituting the total strain modulus by the dynamic modulus of elasticity for the moment when the structure is exposed to seismic impact, the effort is reduced by $2 \%$ (model No.1.3 in Fig.2). The latest design model on an elastic foundation, taking into account the dynamic modulus of elasticity, most accurately reflects the actual behavior of the structure under load.

In a geometrically nonlinear formulation, we additionally approach a more realistic picture, which demonstrates an increase in internal forces of 10 to $20 \%$ in the most loaded columns due to the "accumulation" of the stress-deformed state during the erection from stage to stage (models No.2.1 on a rigid subsoil, No.2.2. on elastic subsoil of total strain modulus and No 2.3. on elastic subsoil of dynamic modulus of elasticity in Fig. 2).

When comparing the values of the two main periods of natural oscillations for each of the six design FEA-models, it is obvious that the history of application of loads does not affect the rigidity of the foundation on the last erection stage. Thus the main influence is due to the type of subsoil model taken and its rigidity (right diagram in Fig. 2).
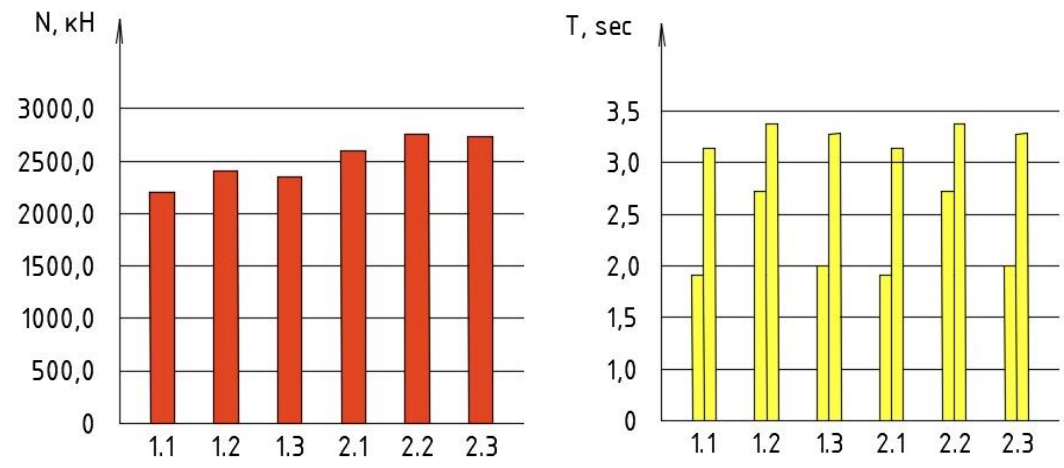

Fig. 2. Comparison of seismic impacts on columns (left) and principal vibration periods (right).

Numerical dynamic FEA design models of structures and subsoils are difficult to perform as any minor deviations from the actual parameters of the constructed object can lead to a significant distortion of the results. In international engineering practice, the most commonly used subsoil model is the Winkler one (Ilyichev et.al, 2016) with a single bed ratio, used by authors in models 1.2, 1.3, 2.2 and 2.3. However, this model is applicable mainly to solving static problems. Detailed subsoil models of solid finite elements or semi-infinite elements have a high complexity, which dramatically increases the risk of making mistakes in the absence of experimental verification of the dynamic parameters of a model with the characteristics of an already erected structure. Therefore, the detailed dynamic models mainly used in scientific practice (Tyapin, 2014). In Russian design code SP 26.13330.2012 "Foundations of Dynamic Machines" and in American one ASCE 4-16 "Seismic Analysis of Safety-Related Nuclear Structures", both implemented for industrial buildings under dynamic effects, a different approach is used, when determining in the center of an absolutely rigid slab foundation the equivalent springs and dampers (Birbraer, 1996; Nuzhdin et.al, 2018). These equivalent parameters of the numerical model can be easily checked instrumentally, using three-component seismic recorders as the American device "REFTEK-130" or the Russian device "REGISTRSD" (Senin et.al, 2017). The authors express their gratitude for the consulting support from one of the developers of the "REGISTR-SD" M.N. Voskresensky, with the help of which the instrumental studies of the considered emergency state seventeen-story residential building were carried out (Fig. 3), which made it possible to clarify the parameters of the dynamic models of the foundation for this study and the entire structure.

In conclusion, it should be noted, that the conducted numerical-analytical study demonstrates the high importance of taking into account the erection history for calculating 
the internal forces of multi-storey reinforced concrete frame buildings. The reflected methodology of a preliminary analytical calculation, can be useful, including for classical linear calculations of buildings and structures, in which the self-weight of the structures has no decisive effect in total combinations of external loads. The instrumental validation of the subsoil dynamic parameters allows designers to get the most accurate Soil-Structure Interaction model and to validate the structural analysis results.
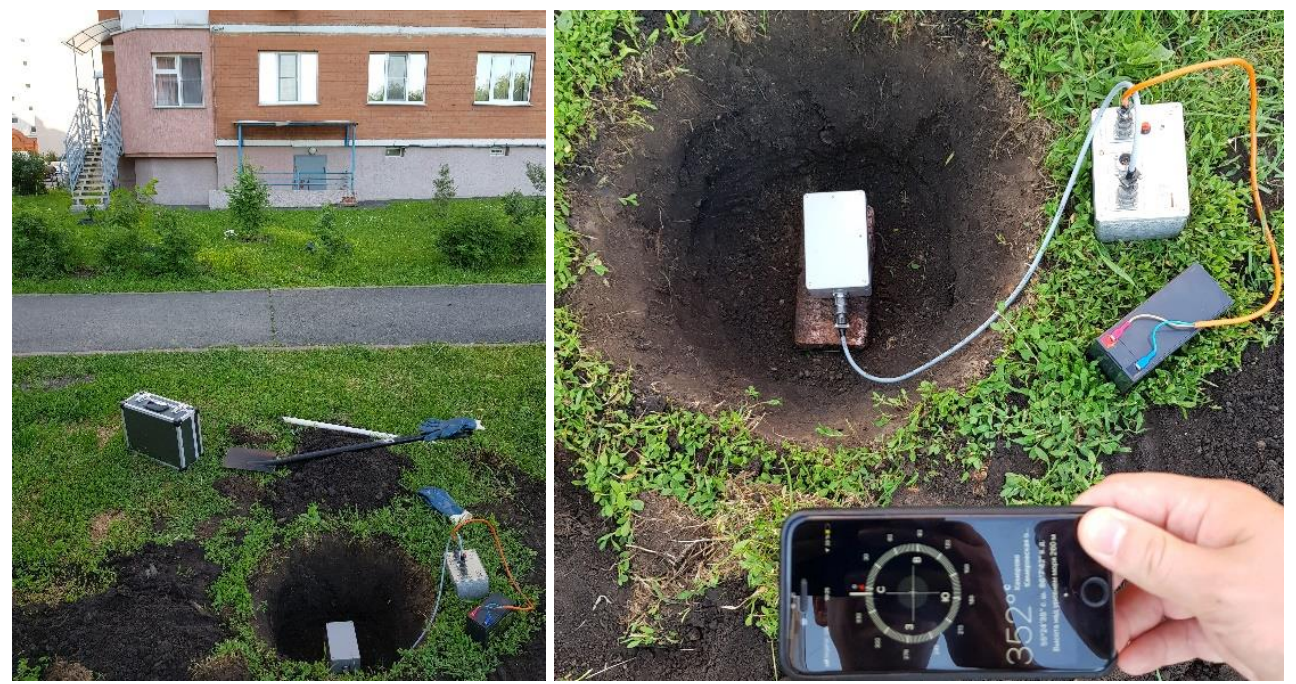

Fig. 3. An example of instrumental determination of the dynamic parameters of the subsoil and of the total structure for the studied residential high-rise building using the "REGISTR-SD".

\section{References}

1. O.G. Kumpyak, Z.R. Galyautdionv, O.R. Pachmurin, V.S. Samsonov, Reinforced concrete and stone structures, 673 (ASV, Mocsow, 2014) (in Russian)

2. A.V. Perelmuter, V.I. Slivker, Analytic model of structures and analysis capability of them, 600 (2011)

3. A.V. Perelmuter, M.A. Mikitarenko, S.G. Burygin, Structural Analysis with Lay-outs Changed during the Action of Load, 251 (1996)

4. A.V. Perelmuter, O.V. Kabantsev, Analysis of structures with varying design scheme, $148(2015)$

5. V.A. Ilyicheva, R.A. Mangusheva, Reference geotechnics. Foundations, foundations and underground structures, 1040 (2016)

6. A.G. Tyapin, Accounting for the interaction of structures with the base in the calculation of seismic effects, 136 (2014)

7. A. N. Birbraer, Calculation of structures for seismic resistance, 255 (1998)

8. L.V. Nuzhdin, V.S. Mikhailov, I.D. Yankovskaya, Geotechnics Fundamentals and Applications in Construction: New Materials, Structures, Technologies and Calculations, 210 (2019)

9. L.N. Senin, T.E. Senina, M. N. Voskresensky, Hardware and software complex "registr-sd" for the study of seismic effects characteristics of objects under the exposure of elastic oscillations. Experiment instruments and technique, 4, 158 (2017) 